\title{
Background estimation and adaptation model with light- change removal for heavily cown-sampled video surveillance signals
}

\author{
Citation for published version (APA): \\ Cvetkovic, S. D., Bakker, P., Schirris, J., \& With, de, P. H. N. (2009). Background estimation and adaptation \\ model with light-change removal for heavily cown-sampled video surveillance signals. In Proceedings of the \\ IEEE International Conference on Image Processing (ICIP 2006), October 8-11, 2006, Atlanta, Georgia (pp. \\ 1829-1832). Institute of Electrical and Electronics Engineers. https://doi.org/10.1109/ICIP.2006.312602
}

DOI:

10.1109/ICIP.2006.312602

Document status and date:

Published: 01/01/2009

\section{Document Version:}

Publisher's PDF, also known as Version of Record (includes final page, issue and volume numbers)

\section{Please check the document version of this publication:}

- A submitted manuscript is the version of the article upon submission and before peer-review. There can be important differences between the submitted version and the official published version of record. People interested in the research are advised to contact the author for the final version of the publication, or visit the $\mathrm{DOI}$ to the publisher's website.

- The final author version and the galley proof are versions of the publication after peer review.

- The final published version features the final layout of the paper including the volume, issue and page numbers.

Link to publication

\footnotetext{
General rights

- You may freely distribute the URL identifying the publication in the public portal. follow below link for the End User Agreement:

www.tue.nl/taverne

Take down policy

If you believe that this document breaches copyright please contact us at:

openaccess@tue.nl

providing details and we will investigate your claim.
}

Copyright and moral rights for the publications made accessible in the public portal are retained by the authors and/or other copyright owners and it is a condition of accessing publications that users recognise and abide by the legal requirements associated with these rights.

- Users may download and print one copy of any publication from the public portal for the purpose of private study or research.

- You may not further distribute the material or use it for any profit-making activity or commercial gain

If the publication is distributed under the terms of Article $25 \mathrm{fa}$ of the Dutch Copyright Act, indicated by the "Taverne" license above, please 


\section{BACKGROUND ESTIMATION AND ADAPTATION MODEL WITH LIGHT-CHANGE REMOVAL FOR HEAVILY DOWN-SAMPLED VIDEO SURVEILLANCE SIGNALS}

\author{
Sascha Cvetkovic * ${ }^{*}$ Peter Bakker*, Johan Schirris* \\ * Bosch Security Systems \\ 5616LW-2 Eindhoven, The Netherlands \\ \{sacha.cvetkovic, peter.bakker, johan.schirris\}@nl.bosch.com
}

\begin{abstract}
This paper describes a background-subtraction system with light change-detection which works on a luminance QCIFsize video signal for surveillance applications. The new proposed pixel background model is controlled by a statistical threshold and is robust for cluttered background and small object motions. Moreover, for light-change detection, we introduce temporal prediction of pixel values to estimate trends while quickly adapting to scene changes to facilitate a very sensitive detection of moving targets. Experiments show that a local contrast enhancement applied prior to downsampling improves detection sensitivity, and combined with the shifted scaled difference and the Wronskian determinant operators provides the best background/foreground detection.
\end{abstract}

Index terms: Background subtraction, light-change, surveillance

\section{INTRODUCTION}

In surveillance applications, the camera has typically a fixed position and for security reasons, system users are particularly interested in the moving regions in the video signals differing from the (possible) camera motion. Background (BG) subtraction is used to segment moving regions in image sequences, by comparing each new frame to a model of the scene BG. In this paper, we present an efficient nonparametric $\mathrm{BG}$ model and a $\mathrm{BG}$-subtraction algorithm that require only the luminance channel of a heavily downsampled video. The model has three advantages. First, the model can handle complex situations where the BG of the scene is cluttered and not completely static but contains small motions, such as found in tree branches and leaves. Second, the model can also extrapolate luminance values to estimate pixel trends and incorporate medium-speed changes, and it adapts quickly to scene changes to facilitate a very sensitive detection of moving targets. Third, it learns objects that are detected as foreground ( $\mathrm{FG}$ ) for a long time and we propose a solution for learning the BG objects starting to move after an initial BG model was created. At the same time, we found a way to reduce memory size significantly.

This paper also shows the effect of heavy down-sampling of the input video to QCIF resolution and the influence of a local image enhancement method (CLAHE) that is applied prior to the down-sampling to circumvent sensitive quality deterioration. Due to the heavy down-sampling of the video signal, image noise does not have a normal distribution anymore, but it behaves like quantization noise which deteriorates the operation of common light-change detectors.

\author{
Peter H.N. de With* \\ * University of Technology Eindhoven/LogicaCMG \\ 5600MB Eindhoven, The Netherlands \\ P.H.N.de.With@tue.nl
}

We present a light-change removal algorithm that uses only the luminance signal and discuss its performance.

The sequel of this paper is organized as follows. Section 2 briefly reviews related work. Sections 3, 4 and 5 comment on some individual system aspects and behavior and introduce corresponding improvements. Section 6 provides results.

\section{RELATED WORK}

Literature provides many BG subtraction algorithms. A good overview and performance comparison is given in [1], where it is explored to solve the challenging demands of realistic surveillance systems: varying levels of illumination during the day as well as exposure control of the camera that is selfreacting to the $\mathrm{FG}$ objects and scene changes, light changes (shadows and light spots), non-static BG objects and multimodal BG (trees, ventilator fans, traffic scenes.), etc. To accommodate real-time requirements, those algorithms should be computationally efficient with low memory requirements.

Most BG-subtraction algorithms learn what is normal in the scene (i.e., construct a BG model) and then detect moving objects by finding image regions that do not conform to the model. This often amounts to modeling the normal behavior at each pixel by a probability distribution, for example a Gaussian or a mixture of Gaussians [2]. A non-parametric model [3] represents the $\mathrm{BG}$ information directly by the observed samples. Most of the algorithms are dealing with full-resolution video signals and use HSV or RGB-color systems for light-change detection, which is often an important part of such systems (see the overview of shadowremoval algorithms in [4]). In [5], an interesting algorithm is presented that operates on the image gradients, but it is not performing sufficiently well for our needs. Finally, in [6], an attractive algorithm is presented that works only on the luminance channel with which we will compare our approach.

\section{BACKGROUND MODEL}

For our research, we adopted a BG model [3] in which the model contains a mixture of distributions with small standard deviation. It contains time-discrete samples $x_{i}$ of previous pixel values and the probability that a new sample $x$ belongs to the $\mathrm{BG}$ is

$P(x)=\frac{1}{N} \sum_{i=1}^{N} \frac{1}{\sqrt{2 \pi \sigma_{i}^{2}}} e^{-\frac{1}{2} \frac{\left(x-x_{i}\right)^{2}}{\sigma_{i}^{2}}}$,

where $\sigma_{i}$ is the standard deviation. If $P(x)>T_{\text {sum }}$, the pixel is classified as BG. The same Gaussian kernel is used for all samples corresponding to a particular pixel, but there can be 
different standard deviations for different pixels. This model stores for each BG pixel a number of sample pairs from which the standard-deviation describing pixel noise is calculated. To adapt to changes in the scene, the BG model is periodically updated. We consider the following aspects.

a) To prevent false negatives, only non-FG pixels and pixels marked as light changes are updated.

b) The model features learning of objects that are detected as $\mathrm{FG}$, but do not move. If a pixel is marked as FG in many successive frames, we perform a "blind" update of that pixel.

c) To cope with BG objects that start to move in a repetitive fashion after the initial BG model was created, or that are cluttered, like trees in a wind, we propose a special mode of $\sigma$ estimation in which all inter-frame differences (including FG pixels) are used. The pixel st. dev. $\sigma$ will increase for those objects and they will be included in the learning.

We base our algorithm on the following steps: (1) process an input frame, (2) perform $\mathrm{BG}$ subtraction, and (3) mark pixels as FG, BG or light change. Further morphological processing (noise removal, close operation) improves the FG, which is grouped into components. For each component, (4) find the bounding box, (5) count the number of pixels, and (6) find the center of the mass. The detected components are then passed to the tracking module and a unit for higher semantic conclusions.

\section{IMPROVEMENTS OF THE BACKGROUND- SUBTRACTION ALGORITHM}

\subsection{Calculation of standard deviations}

In addition to samples $x_{i}$ in Eq. (1), we need to obtain the standard deviation $\sigma$ for each pixel. As already presented in [3], the standard deviations are estimated from variations in successive frames: a histogram of absolute differences $\left|x_{i}-x_{i+1}\right|$ of pixels from BG memory is constructed and the median $m$ of the histogram is found. Consequently, $\sigma=m / 0.68 \sqrt{2}$.

In our application, due to the heavy down-sampling of the video, the noise is not described by a Gaussian distribution $\mathrm{N}\left(0, \sigma^{2}\right)$, but rather acts as quantization noise. In many cases, the absolute differences $\left|x_{i}-x_{i+1}\right|$ are zero, while the pixel luminance value slowly drifts. Use of the median value will result in $\sigma=0$ for almost all pixels in the scene. We estimated $\sigma$ as an average of the absolute differences $\left|x_{i}-x_{i+1}\right|$. Very large differences can be ignored, to avoid the influence of $\mathrm{FG}$ objects in the $\sigma$ model. If $n$ BG memory pairs exist, $2 n-1$ consecutive abs. diff. pairs are used as an initial $\sigma$ estimate.

To detect FG regions in the current frame, we evaluate Eq.(1) for each pixel. Usually, Eq. (1) is implemented as a Look-Up Table (LUT). Since threshold $T_{\text {sum }}$ is unknown, we have to cover all possible values of the differences $\left|x-x_{i}\right|$ (at 8bit resolution) and $\sigma$ (having typically a maximum value of 8 with 5-bit precision). Therefore, the LUT size is 256x32, where each element has 16-bit precision. Instead of this approach, we propose to incorporate the value of the threshold in Eq. (1). We noticed that for a provisional value of the $T_{\text {sum }}$, large portions of the LUT are not used because the related probabilities are several orders of magnitude smaller or larger than $T_{\text {sum }}$. Therefore, it is only interesting to relate the threshold $T$ to the value of the difference $\Delta_{t}=\left|x-x_{i}\right|$, normalized with $\sigma$. Only by deriving $\sigma$, we can obtain the differences that should not be neglected in relation to a certain $T$. Hence, we define the middle reference point of the Gaussian function equal to the value of the threshold $T$, resulting in

$P(x)=\frac{1}{N} \sum_{i=1}^{N} f(x)$, where $f(x)=\left\{\begin{array}{cc}1 & \text { if } \frac{\Delta_{i}}{\sigma_{i}}-T<0, \\ e^{-\frac{1}{2}\left(\frac{\Delta_{i}}{\sigma_{i}}-T\right)^{2}} & \text { otherwise. }\end{array}\right\}$

If $P(x)>T_{\text {sum }}$, the pixel is classified as BG. Threshold $T_{\text {sum }}$ offers a large system flexibility, since we can specify how many pixels from the $\mathrm{BG}$ memory have to be close (or equal) to the current pixel value to mark that pixel as BG. With $T=$ 0, Eq. (2) is performing equivalent to Eq. (1). Notice that we do not use the normalization part of the Gaussian function, otherwise e.g. value $\Delta_{t}=0$ would yield a different probability for the different values of $\sigma$.

Let us provide an example. If we want to tolerate pixel deviations of about $2 \sigma$ around a pixel mode, we can define $T=2$. If the difference $\Delta_{t}$ is larger than $2 \sigma$, the pixel will be marked FG unless the total sum of contributions from other BG memories is larger than $T_{\text {sum }}$. For the same accuracy, our solution needs a LUT of $11 \times 32$ entries of 6-bit numbers, which is almost 24,000 times less than the standard LUT size.

\subsection{Two thresholds for the foreground detection}

In the conventional approach, if the estimated probability $P(x)>T$, it is sufficient to mark that pixel BG. We observed that when having a single detection threshold, the designer has to make a tough compromise between false positives and false negatives with doubtful results. Instead, we introduce a second threshold and propose the following upgrade of the FG detection.

a) One large and one small threshold are used. Pixels that are marked FG by detection with a larger threshold are having a larger probability to belong to the real FG. Consequently, they are assigned a higher weight in the FG mask and vice versa. $\mathrm{FGM}_{\mathrm{i}}$ represents the initial $\mathrm{FG}$ mask.

$$
F G M_{i}=\left\{\begin{array}{cc}
w_{1}, & \text { if pixel }=F G \wedge T=T_{\text {large }} \\
w_{2}<w_{1}, & \text { if pixel }=F G \wedge T=T_{\text {small }}
\end{array}\right\} .
$$

b) In the process of noise filtering, a square 2-D FIR filter of size $n X n$ is applied to the initial FG mask (the symbol $\otimes$ represents 2-D convolution). The current pixel is then classified as FG if the filter output is larger than a certain threshold ( $F G M$ is a noise-filtered FG mask). In short, instead of having only one threshold, we also consider the neighborhood of the pixel and -with more confidencedetermine from the surrounding context whether the current pixel is a real FG pixel. More formally, this is noted as

$F G M=\left[\left(B_{0} x_{n} \otimes F G M_{i}\right) \geq T_{\text {noise }}\right]$. 


\section{HANDLING LIGHT CHANGES}

During daytime, many changes in lighting occur which can have drastic effects on the scene appearance, especially when combined with auto-gain/auto-iris mechanisms of the camera. Naturally, these influences should be ignored, as they do not represent real alarms. We have constructed three mechanisms dealing with light changes. If a global change occurs, causing the majority of image pixels to be marked as FG, we reinitialize the model to quickly adapt to the new scene. However, local light changes (e.g. caused by the sun shining into a part of the room through a window, or shadows of persons) are handled by comparing the structure of regions of a reference image $\left(r_{t}=\alpha \cdot r_{t-1}+(1-\alpha) \cdot x_{t}\right.$, where $r$ refers to the reference image, $x$ the current input image, $t$ for time). Local light changes typically make a region brighter or darker than a reference image without changing its gray level structure. Finally, to handle medium speed changes, the model incorporates linear predictors.

\subsection{Local light changes}

One of the standard approaches (see e.g. [6]) that works only on the luminance signal calculates the Normalized Cross Correlation (NCC) between input pixel $x$ and the reference image $r(\bar{r}$ and $\bar{x}$ are local averages of a pixel value in the $n \times n$ surrounding window). This cross correlation is defined by

$N C C=\sum_{i=1}^{n^{2}}\left(x_{i}-\bar{x}\right)\left(r_{i}-\bar{r}\right) /\left(\sqrt{\sum_{i=1}^{n^{2}}\left(x_{i}-\bar{x}\right)^{2}} \cdot \sqrt{\sum_{i=1}^{n^{2}}\left(r_{i}-\bar{r}\right)^{2}}\right)$.

However, for regions with little or no texture (such as a blank wall), the NCC essentially compares noise and does not yield good results. To deal with non-textured planes, the Shifted Scaled Difference (SSD) was introduced. The idea is that the luminance change of the average value is almost identical to the luminance change of the actual pixel. One of the problems is that the SSD assumes a luminance shift that should be approximately equal for all the pixels in the neighborhood. In reality, not all pixels from the $n \times n$ neighborhood are involved in the light change, and moreover, they also do not have an equal shift. This results from various factors, like downsampling (which is a low-pass filter), small object sizes (in pixels), various overlapping shadow directions from several light sources, etc. Hence, we propose to modify the SSD formula, which is introduced to cope with mixing of the FG and the BG objects when applying the down-sampling, so that $S S D_{d}=\sum \frac{\left|x_{i}-r_{i}-k(\bar{x}-\bar{r})\right|}{\sigma_{i}+\varepsilon}$, with $k<2$ and $\varepsilon=0.1$.

During our experiments, we found that the NCC measurement does not work well for the down-sampled input video, due to the large loss of details (local standard deviation is often close to zero) and noise that is not behaving as white noise. Instead, we used the Wronskian test [7] for determining the linear dependence between vectors ( $n \times n$ pixel window). It is shown that if the Wronskian determinant (Wron) is equal (or close) to zero, two vectors are linearly dependent, thus
Wron $\left(\frac{x}{r}\right)=\frac{1}{n} \cdot \sum_{i=1}^{n} \frac{x_{i}^{2}}{r_{i}^{2}}-\frac{1}{n} \cdot \sum_{i=1}^{n} \frac{x i}{r i}=0$.

If $r$ represents the reference $\mathrm{BG}$ image and $x$ the input image, Wron $(x / r)$ detects shadows and Wron $(r / x)$ detects bright light changes. In both cases, Wron should be between zero and a small threshold $T_{l c}>0$.

Finally, we obtain the initial light change $L C_{i}$ by specifying that $L C_{i}=$ Wron $+S S D_{d}$.

We also propose to add high-level logic on top of Wron and $S S D_{d}$. Local light changes typically do not change the gray-level structure of the image, which we check by looking at the local standard deviation of the input image $\left(\sigma_{x}\right)$ and the reference image $\left(\sigma_{r}\right)$ and we create light change mask $L C M$. For darker areas of the video, we use the relative change to increase its sensitivity, so that

$$
L C M=\left\{\begin{array}{c}
1 \quad \text { if } L C_{i}=1 \wedge\left(\left|\sigma_{x}-\sigma_{r}\right| / \sigma_{r}<T_{\operatorname{det} a i l} \wedge Y<T_{\text {lum }}\right) \\
\vee\left(\left|\sigma_{x}-\sigma_{r}\right|<T_{\text {det } a i l} \wedge Y \geq T_{\text {lum }}\right), \\
\text { otherwise. }
\end{array}\right\}
$$

\subsection{Linear temporal predictors}

To reduce the effect of medium-speed global light changes, we include linear temporal predictors to attempt the prediction of trends in the pixel value. We perform a linear extrapolation of the most recent BG model samples $x_{t-3}, x_{t-2}$, $x_{t-1}$ and $x_{t}$. The BG model is updated two frames at a time, so samples $x_{t-1}$ and $x_{t}$ come from successive frames and their difference is most likely noise and cannot be used to predict trends.

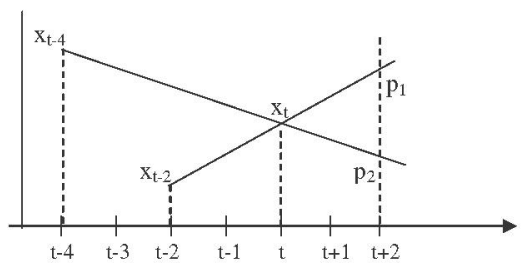

Fig. 1. Linear temporal predictors for pixel values.

The interval between samples $x_{t-2}$ and $x_{t}$ is longer and it corresponds to the model update time. We therefore skip every other value and calculate predictions of the pixel value at time $t+2$ (see Fig. 1) by:

$$
p_{1}=2 x_{t}-x_{t-2}, \quad p_{2}=0.5\left(3 x_{t}-x_{t-4}\right) .
$$

Note that we are not limited to two predictors, since we could also generate $p_{3}$ based on $x_{t-6}$ and $x_{t}$, and so on. These frames, now filtered by the BG mask (as it is from another time moment) are stored in the BG memory.

\section{RESULTS AND CONCLUSIONS}

We have evaluated the proposed model using both in- and out-door sequences and show results of a crossing observed during the night and day (Figs. 2 and 3). At the top-left corner of these figures, the original image is shown. FG remaining after light-change detection is depicted as white. The light- 
changes are: SSD (blue), NCC or Wron (red) and SSD\&Wron or SSD\&NCC (yellow).

Numerous light-changes are detected, either originating from headlights (Fig. 2) or the sun (shadows in Fig. 3). It can be seen that the use of light-change detection is compulsory in such extreme conditions to cope with false positives.

Down-sampling generally improves the performance of light-change detection (less false positives), especially for SSD. However there is a limit to that since local contrast can be significantly lowered due to the down-sampling which often can lead to detection of FG objects as light changes or complete miss-detection of FG objects (the FG detection works better on original resolution images).

Applying local contrast enhancement prior to downsampling sometimes increases the sensitivity of the FG detection and contributes to the overall result especially for small low-contrast FG objects, which otherwise would be detected as a light-change (bicycle at bottom-right corner of Fig. 2). We have achieved similar results (Fig. 2 bottom right, LCM) using the standard-deviation test of Eq. (8). Therefore it is worthwhile to further test these kinds of techniques, especially their influences on the detection sensitivity and change of the image noise model as captured in the $\sigma$ memory.

Often, the performance is a compromise between merging FG objects, the amount of false positives and the false negatives. Thresholds have different operational values in the different situations and resolutions. Down-sampled images usually require a higher threshold for sensible light-change detection.

Although less complex, the performance of the Wron change detector is comparable to NCC. On down-sampled input with large shadows, Wron performs better than NCC. Multi-component light-change detection methods as for instance described in [4] are often computationally more expensive and also presume existence of 3-component data, which is a "luxury" we do not have. At the same time, our image resolution is QCIF- again much lower than what is usually the case. Concerning all the discussed limits, we

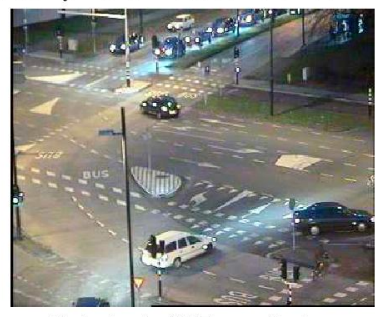

Original, CIF resolution

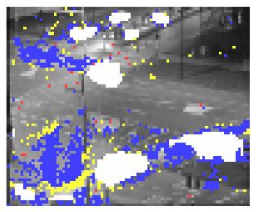

QCIF

SSD \& Wron

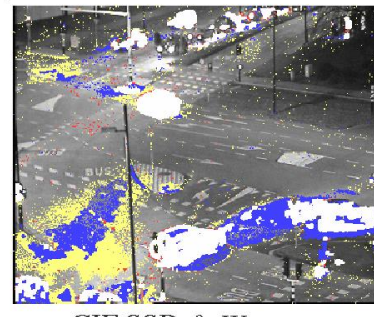

CIF SSD \& Wron

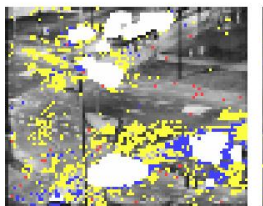

QCIF, CLAHE SSD \& Wron

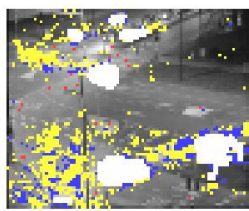

QCIF, SSD, NCC \& LCM
Fig. 2. Results of FG and light-change detection on headlights.

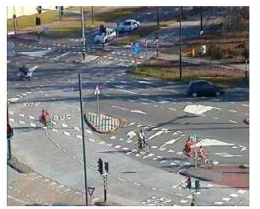

Original, CIF res

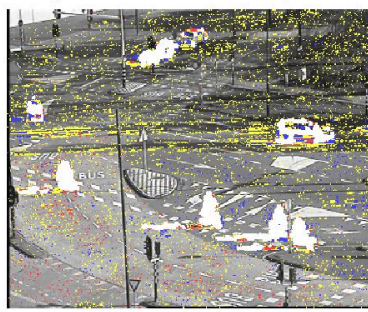

CIF SSD \& NCC

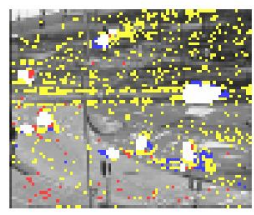

QCIF SSD \& NCC
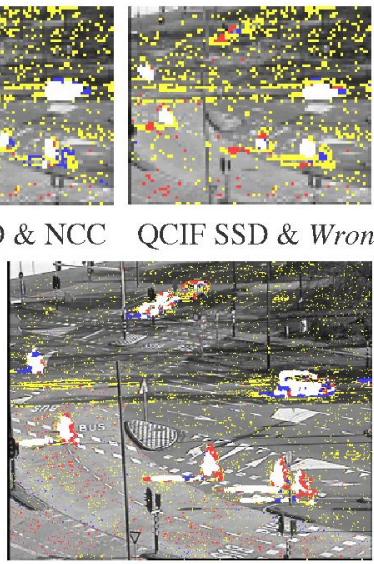

CIF SSD \& Wron
Fig. 3. Results of FG and light-change detection on shadows.

succeeded in making a system that can still work with a decent performance on such a dataset in real time $(25 \mathrm{fr} / \mathrm{s}$ progressive, CIF/QCIF format, luminance channel only). Other systems [1],[4] cannot work as good in the same conditions with all the functionality required. However, the performance of the light-change detection is not always satisfactory: there are always exceptional scenes and light conditions in which real FG objects are taken out or real light changes are missed. Continuous improvement is required.

\section{REFERENCES}

[1] S.-C. S. Cheung and C. Kamath, "Robust techniques for background subtraction in urban traffic video," Proc. SPIE Electr. Imaging, VCIP 2004, San Jose, Vol. 5308 A, pp.881-892, Jan. 2004.

[2] C. Stauffer and W.E.L. Grimson, "Adaptive background mixture models for real-time tracking," IEEE Proc. CVPR'99, Vol. 2, pp. 246-252, 1999

[3] Elgammal, A, Duraiswami, R, Harwood, and D., Davis, L.S., "Background and foreground modeling using nonparametric kernel density estimation for visual surveillance," Proceedings of the IEEE, Vol. 90, Issue 7, pp. $1151-1163$, July 2002.

[4] Prati, I. Mikic, M. Trivedi, and R. Cucchiara, "Detecting moving shados: algorithms and evaluation," IEEE Trans. Pattern Analysis and Machine Intelligence 25, pp. 918-923, July 2003.

[5] Bevilacqua, A., di Stefano, L., Lanza, A., and Capelli, G., "A Novel Approach to Change Detection Based on a Coarse-to-Fine Strategy", IEEE Proc. Int. Conf. Image Proc. (ICIP 2005), Genova (Italy), 11-14 Sept. 2005, Vol. 2, pp. 434 - 437, Sept. 2005.

[6] T. Brodsky, "Discriminating between changes in lighting and movement of objects in a series of images using different methods depending on optically detectable surface characteristics," U.S. Patent 2003/0194110, April 16, 2002.

[7] Durucan, E. and Ebrahimi, T, "Change detection and background extraction by linear algebra," Proceedings of the IEEE, Vol. 89, Issue 10, pp. 1368 - 1381, Oct. 2001. 